\title{
Identification of Epigenetic Mechanisms Involved in the Anti-Asthmatic Effects of Descurainia sophia Seed Extract Based on a Multi-Omics Approach
}

\author{
Su-Jin Baek ${ }^{1}$, Jin Mi Chun ${ }^{2}$, Tae-Wook Kang ${ }^{1}$, Yun-Soo Seo ${ }^{2}$, Sung-Bae Kim ${ }^{2}$, Boseok Seong ${ }^{3}$, \\ Yunji Jang ${ }^{3}$, Ga-Hee Shin ${ }^{1}$ and Chul Kim ${ }^{3, * \mathbb{D}}$ \\ 1 Bioinformatics Group, R\&D Center, Insilicogen Corporation, 35, Techno 9-ro, Yuseong-gu, Daejeon 34027, \\ Korea; sjbaek@insilicogen.com (S.-J.B.); twkang@insilicogen.com (T.-W.K.); ghshin@insilicogen.com (G.-H.S.) \\ 2 Herbal Medicine Research Division, Korea Institute of Oriental Medicine, 1672 Yuseong-daero, Yuseong-gu, \\ Daejeon 34054, Korea; jmchun@kiom.re.kr (J.M.C.); sys0109@kiom.re.kr (Y.-S.S.); suaa10@kiom.re.kr (S.-B.K.) \\ 3 Future Medicine Division, Korea Institute of Oriental Medicine, 1672 Yuseong-daero, Yuseong-gu, \\ Daejeon 34054, Korea; sungbosal@kiom.re.kr (B.S.); jangbing@kiom.re.kr (Y.J.) \\ * Correspondence: chulnice@kiom.re.kr; Tel.: +82-42-868-9582
}

Received: 15 October 2018; Accepted: 30 October 2018; Published: 5 November 2018

\begin{abstract}
Asthma, a heterogeneous disease of the airways, is common around the world, but little is known about the molecular mechanisms underlying the interactions between DNA methylation and gene expression in relation to this disease. The seeds of Descurainia sophia are traditionally used to treat coughs, asthma and edema, but their effects on asthma have not been investigated by multi-omics analysis. We undertook this study to assess the epigenetic effects of ethanol extract of D. sophia seeds (DSE) in an ovalbumin (OVA)-induced mouse model of asthma. We profiled genome-wide DNA methylation by Methyl-seq and characterized the transcriptome by RNA-seq in mouse lung tissue under three conditions: saline control, OVA-induced, and DSE-treated. In total, 1995 differentially methylated regions (DMRs) were identified in association with anti-asthmatic effects, most in promoter and coding regions. Among them, 25 DMRs were negatively correlated with the expression of the corresponding 18 genes. These genes were related to development of the lung, respiratory tube and respiratory system. Our findings provide insights into the anti-asthmatic effects of $D$. sophia seeds and reveal the epigenetic targets of anti-inflammatory processes in mice.
\end{abstract}

Keywords: Descurainia sophia (D. sophia) seeds; ovalbumin-induced mouse; asthma; DNA methylation

\section{Introduction}

Asthma is a chronic inflammatory and multi-causal disease determined by various genetic, epigenetic and environmental factors [1,2]. In recent years, epigenetic marks have emerged as a potential mechanism for asthma [3-6], and have been shown to regulate many immune cell processes involved in the disease. In addition, several epigenomics and transcriptomics studies have identified DNA methylation as a biomarker for asthma [7-9].

Dietary phytochemicals are not only a rich source of minerals, vitamins and micronutrients, but also contain bioactive components such as phenols, phenolic acids, flavonoids and flavonoid glycosides. These bioactive compounds exhibit great potential against many diseases [10,11], including asthma [12]. Descurainia sophia Webb ex Prantl, which contains several such bioactive compounds [13,14], is one of the original species of Descurainia Sophia (L.) Webb ex Prantl [15]. The seeds of this plant have been used traditionally to treat diseases and symptoms such as cough, asthma and edema $[16,17]$.

Although $D$. sophia seeds have not been extensively investigated, recent studies have revealed the major active components and their effects. For instance, ethanol extract and volatile oil of $D$. sophia 
seeds inhibit the growth of various cancer cell lines in vitro $[16,18,19]$. Several constituents isolated from these seeds exert cytotoxic and anti-inflammatory effects on both human cancer cell lines and murine macrophages [17]. Based on their potential therapeutic effects, these components are predicted to be useful in the treatment of allergies and inflammatory lung diseases. Although the anti-asthmatic effects of $D$. sophia seeds have been partially characterized, to date no study has taken an integrated multi-omics approach to investigate the therapeutic mechanisms underlying their target pathways in asthma. To obtain insight into the epigenetic mechanisms of the anti-asthmatic effects of $D$. sophia seed extract (DSE) in an ovalbumin (OVA)-induced mouse model of asthma, we performed Methyl-seq and RNA-seq to profile genome-wide DNA methylation and gene expression, respectively. In addition, we performed an integrated analysis using epigenomic and transcriptomic data sets (Figure S1). The resultant integrated network of DNA methylation and expression revealed epigenetically regulated genes associated with anti-asthmatic effects.

\section{Results}

\subsection{DSE Treatment Reduces Asthmatic Inflammation in an OVA-Induced Mouse Model}

We collected samples from mice subjected to three treatments: saline (control, $n=3$ ), OVA (asthma-induced mice; $n=3$ ) and DSE (herbal treatment; $n=4$ ) (Figure S2). To evaluate the anti-asthmatic effects of DSE, we monitored the phenotypes of allergic lung inflammation, including histopathological features, the number of infiltrated cells and cytokine expression. First, to evaluate the effects of DSE on lung inflammation in asthma, we sectioned the lungs and stained the sections with hematoxylin-eosin (H\&E). Infiltration of immune cells around blood vessels was higher in OVA-induced mice than in saline-treated mice, but lower in DSE-treated mice (Figure 1A). Next, to confirm that DSE inhibits inflammatory cell infiltration, we counted inflammatory cells, including eosinophils, neutrophils and macrophages, in bronchoalveolar lavage fluid (BALF). In OVA-induced mice, both total inflammatory cells and individual categories of cells (eosinophils, neutrophils and macrophages) were more abundant than in the saline control group, whereas DSE-treated mice had significantly fewer inflammatory cells than untreated asthmatic mice (Figure 1B-E). Furthermore, the levels of interleukin (IL)-4 were significantly elevated in asthmatic mice, but significantly reduced in DSE-treated mice (Figure 1F). IL-4, a type 2 cytokine, plays a key role in the pathogenesis of allergic asthma [20], and IL-4 levels are a major marker of type 2 helper T cells and allergic inflammation. These results confirmed that DSE inhibited OVA-induced allergic lung inflammation by decreasing inflammatory cell infiltration and production of the Th2 cytokine IL-4 in the lung.

\subsection{Methyl-Seq Reveals Distinctive DNA Methylation Changes among Saline-Treated, OVA-Induced and DSE-Treated Mice}

We performed DNA methylome profiling in samples from the same animals to identify differentially methylated regions (DMRs) among the three groups. For all CpGs with a minimum read depth 20, as determined by Methyl-seq, we observed a bimodal distribution of DNA methylation (Figures S3 and S4). We then performed principal component analysis (PCA) on the genome-wide methylation dataset, as shown in Figure 2A. Approximately 5.2\% (23,154 bins) of hyper methylated and $2.75 \%(12,247$ bins) of hypo methylated DMRs (Diff $\geq 10 \%$ and $q$-value $<0.01)$ were selected from a total of 445,288 bins in OVA-induced samples vs. controls (Figure 2B and Table S1), whereas 1.07\% (4818 bins) of hyper methylated and $0.97 \%$ (4379 bins) of hypo methylated DMRs were selected from a total of 449,539 bins in DSE-treated vs. OVA-induced samples (Figure 2C and Table S2). 
A

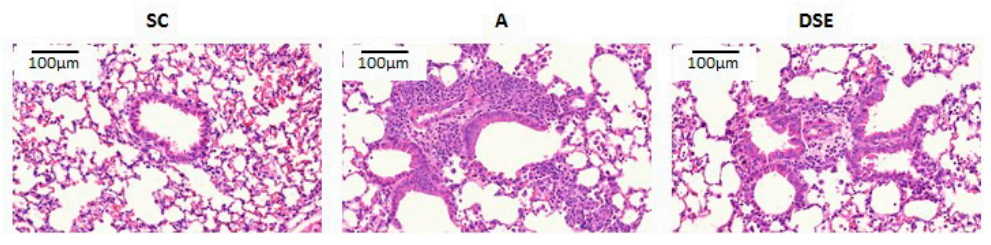

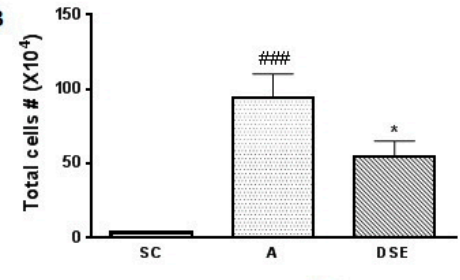

D

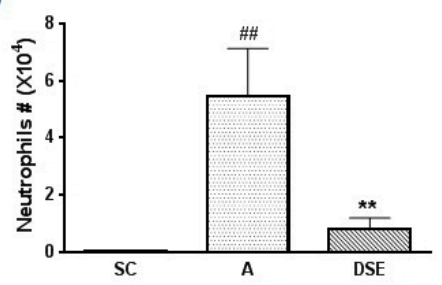

E

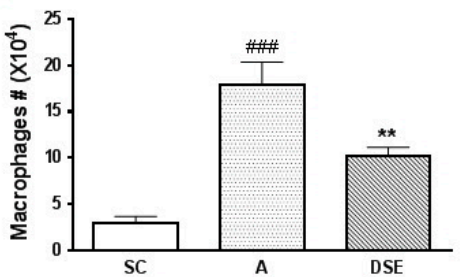

$\mathrm{C}$

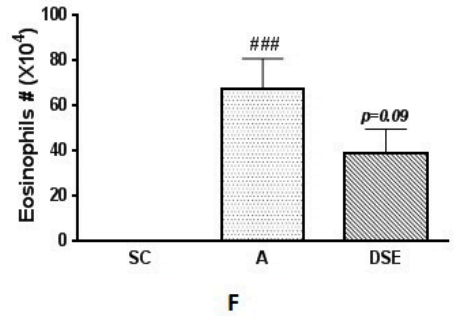

Figure 1. Effects of DSE on phenotypes of allergic asthma in OVA-induced mice. Representative photographs of lung sections stained with H\&E (magnification, $\times 200)($ A). Counts of: total cells (B); eosinophils (C); neutrophils (D); and macrophages (E) in infiltrated BALF. Levels of IL-4 in BALF determined by ELISA (F). Data are presented as means $\pm \operatorname{SEM}(n=7)$. \#\# $p<0.01$, \#\#\# $p<0.001$ compared with the saline control group. ${ }^{*} p<0.05,{ }^{* *} p<0.01$ in allergic lung inflammation vs. vehicle group. SC, Saline Control; A, OVA; DSE, D. sophia seed extract.

Overall, DMRs tended to be detected in functional regions of the genome, such as promoters and coding regions. In OVA-induced samples vs. controls, hyper methylated regions were more frequent than hypo methylated regions in promoter, exon, intron and intergenic regions. By contrast, in the comparison of DSE-treated vs. OVA-induced samples, DMRs did not differ significantly between hyper and hypo methylated regions, except in promoter regions (Figure 2D).
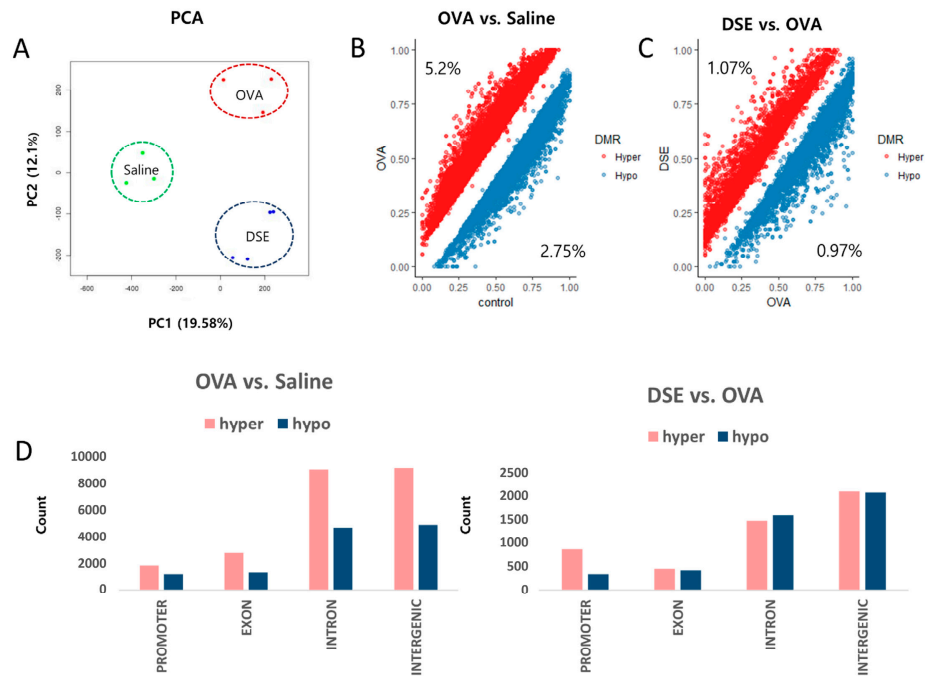

Figure 2. Genome-wide methylation profiling in DMRs. PCA plot of DNA methylation data. Samples are colored according to the experimental group (A). Scatter plot of DMRs in OVA vs. saline control (B). Scatter plot of DMRs in DSE vs. OVA (C). Distribution of DMRs in OVA vs. control (left), and DSE vs. OVA (right) (D). 
We focused on methylation patterns that differed between saline-treated and OVA-induced mice, and were restored to the control level by DSE treatment, i.e., hyper methylated in OVA vs. saline and hypo methylated in DSE vs. OVA ("hyper/hypo"; Figure 3A and Table S3) or hypo methylated in OVA vs. saline and hyper methylated in DSE vs. OVA ("hypo/hyper"; Figure 3B and Table S3). These DMRs, which were identified as key genes, were used in the next step to infer the mechanism underlying the effects of the herbal treatment. We then used the DAVID resource to examine the functional annotation of genes (Figure 3C,D). Genes with the hyper/hypo pattern shown in Figure 3A were enriched in KEGG pathway such as olfactory transduction ( $p$-value: $5.11 \times 10^{-23}$; Calm1, Cnga3, Prkacb, and Prkg1), cytokine-cytokine receptor interaction ( $p$-value: $1.3 \times 10^{-8} ; \mathrm{Hgf}, \mathrm{Flt}, \mathrm{Prlr}, \mathrm{Tgfbr1}$, Kit, Cntfr, Acvrl1, Egfr, Il2Rb, Ifnar2, Crlf2, and Il7), and chemokine signaling pathway ( $p$-value: $2.3 \times 10^{-7} ; A d c y 2$, Prkacb, Raf1, Prkcz, Pik3ca, Elmo1, and Pik3r1; Figure 3C and Table S4). Conversely, genes with the hypo/hyper pattern shown in Figure $3 \mathrm{~B}$ were enriched in functions related to cytokine-cytokine receptor interaction ( $p$-value: $9.58 \times 10^{-7} ; \mathrm{Csf3r}$, Vegfa, Ccl28, Il7r, Ifng, and Crlf2) and natural killer cell mediated cytotoxicity ( $p$-value: $1.2 \times 10^{-4}$; Nfat5 and Ifng; Figure 3D and Table S5).

A

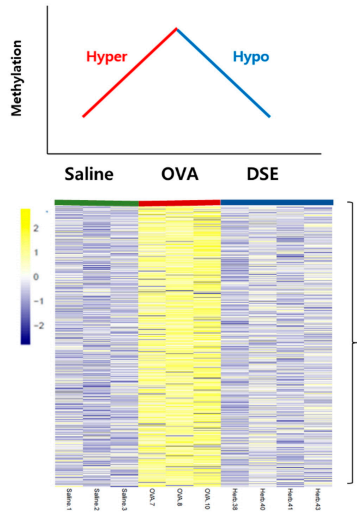

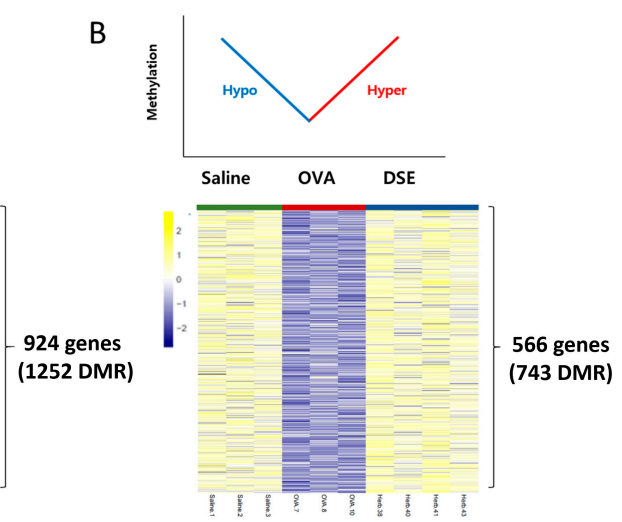

$\mathrm{D}$
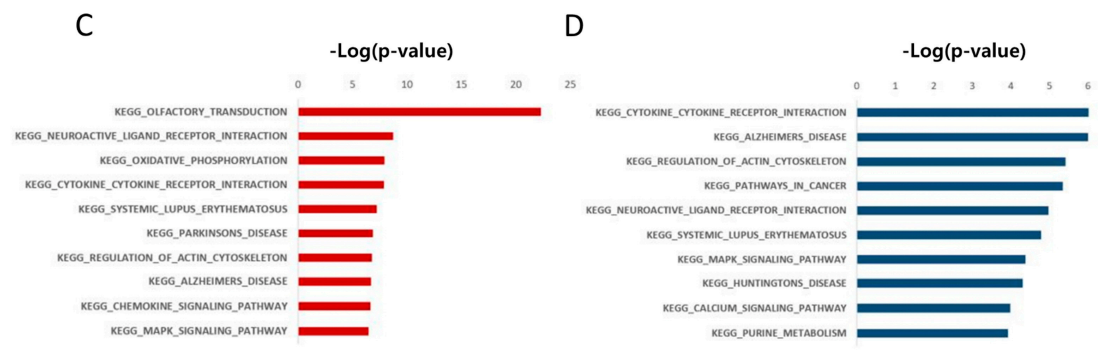

Figure 3. Anti-asthmatic methylation patterns in response to DSE treatment. Heat map of hyper/hypo methylated genes in OVA vs. saline and DSE vs. OVA (A). Heat map of hypo/hyper methylated genes under the same conditions (B). Top 10 functional annotation results of hyper/hypo methylation pattern, consisting of 924 genes (1252 DMRs) (C), and hypo/hyper methylation pattern, containing 566 genes (743 DMRs) (D). KEGG pathway names are shown on the left, and the bars on the right represent the $-\log (p$-value) of the corresponding pathway.

2.3. Identification by Multi-Omics Analysis of Epigenetically Regulated Genes that Were Restored to Control Levels by DSE Treatment

To identify genes regulated by DNA methylation in anti-asthmatic processes, we examined the RNA-seq data for negative correlations between DNA methylation and gene expression. We characterized two distinct patterns: Pattern A, including 17 genes that were downregulated in OVA vs. saline and upregulated in DSE vs. OVA ("down/up") and hyper/hypo methylated (Figure $4 \mathrm{~A}$ and Table 1), and pattern B, consisting of only a single gene that was upregulated in OVA vs. saline and downregulated in DSE vs. OVA ("up/down") and hypo/hyper methylated (Figure 4B and 
Table 1). Both patterns exhibited a negative correlation between DNA methylation and gene expression (Figure S5). In functional terms, these 18 genes were enriched in GO biological process terms pertaining to development of the lung alveolus ( $p$-value: 0.001; Vegfa, Hopx, and Errfi1), lung vasculature ( $p$-value: 0.007; Vegfa and Errfi1), and lung (p-value: 0.017; Vegfa, Hopx, and Errfi1; Table S6).

Pattern A

A
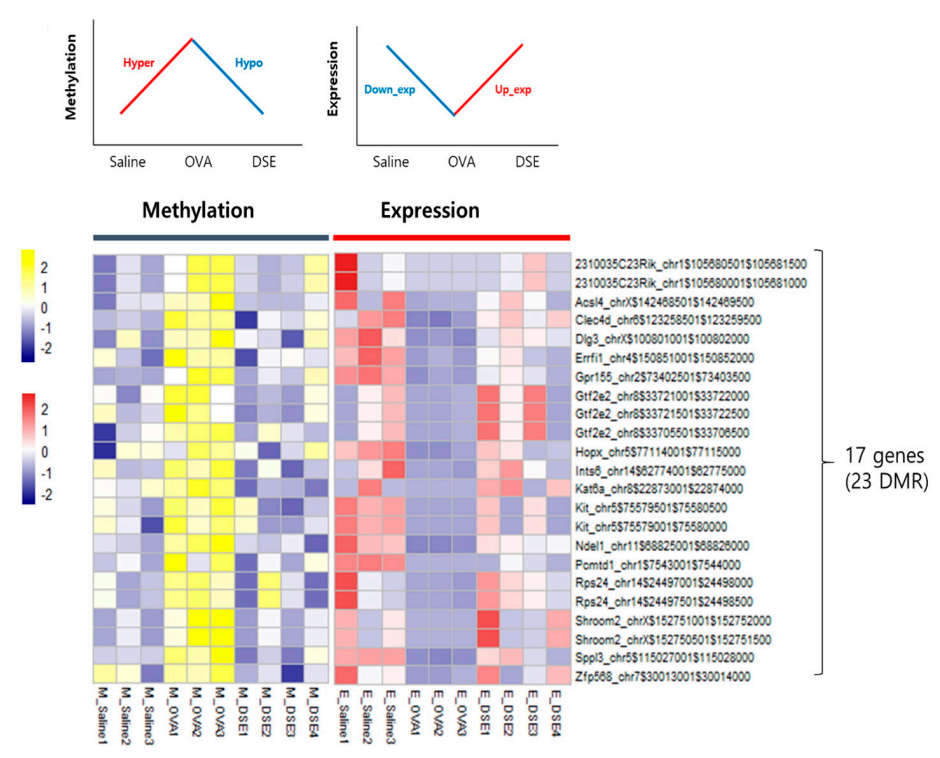

Pattern B

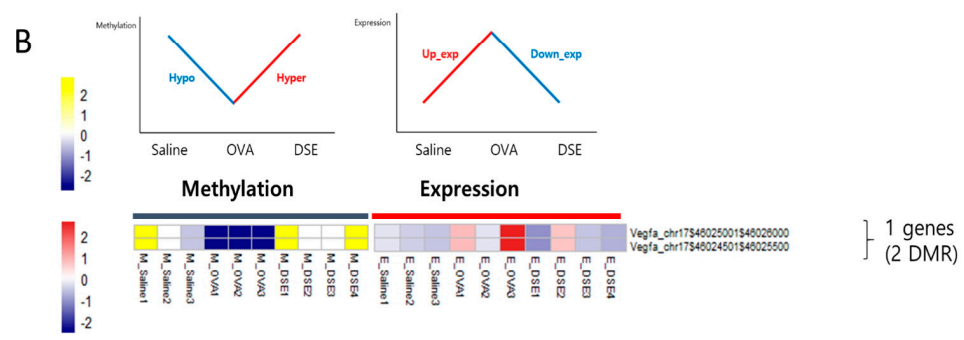

Figure 4. Anti-asthmatic pattern genes with inverse correlation between methylation and expression. Matched heat map of DNA methylation and gene expression profiling in hyper/hypo methylated and down/upregulated genes (A). Matched heat map of DNA methylation and gene expression in hypo/hyper methylated and up/downregulated genes (B).

\subsection{Identification of Two Main Anti-Asthma Genes by Integrated Network Analysis}

To understand the interactions among the anti-asthmatic genes, we performed integrated network analysis of DNA methylation and gene expression during recovery by DSE treatment. Using the 18 genes detected in the pattern analysis described above, we examined the interactions using Ingenuity Pathway Analysis (IPA) software and found 60 network-eligible genes and chemicals associated with asthma (Table S7). To determine the effects of methylation changes, we overlaid the DNA methylation values on the network. Figure 5 shows the predicted pathways related to anti-asthma genes in OVA vs. saline and DSE vs. OVA. In OVA vs. saline, Vegfa was upregulated, whereas Kit, Acsl4, Hopx, Rps24, Errfi1, Gtf2e2, Ints6, 2310035C23Rik (KIAA1468) and Sppl3 were downregulated. Methylation changes were in the opposite direction from expression level changes, as shown in Figure 5A (bar plots next to genes). 

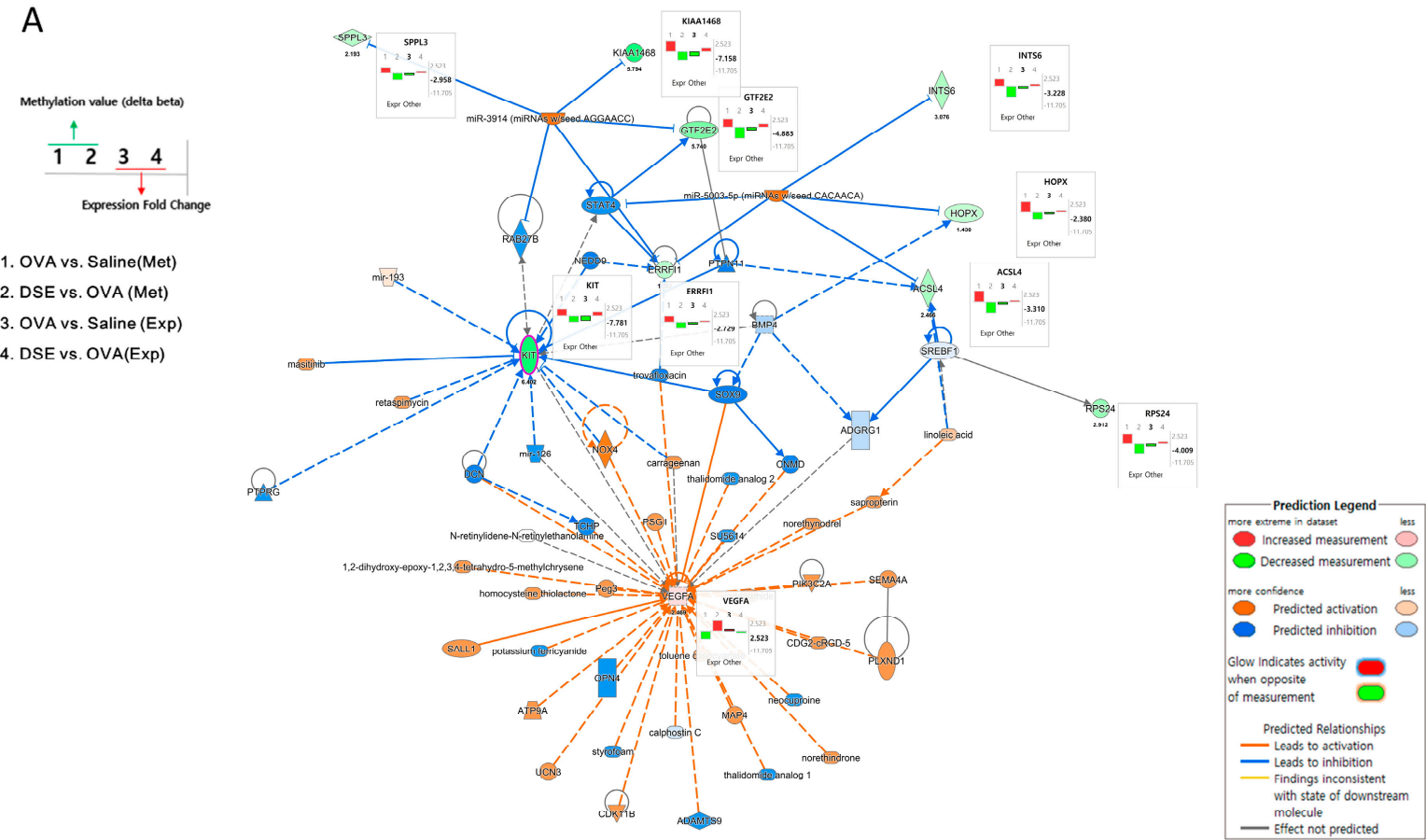

B
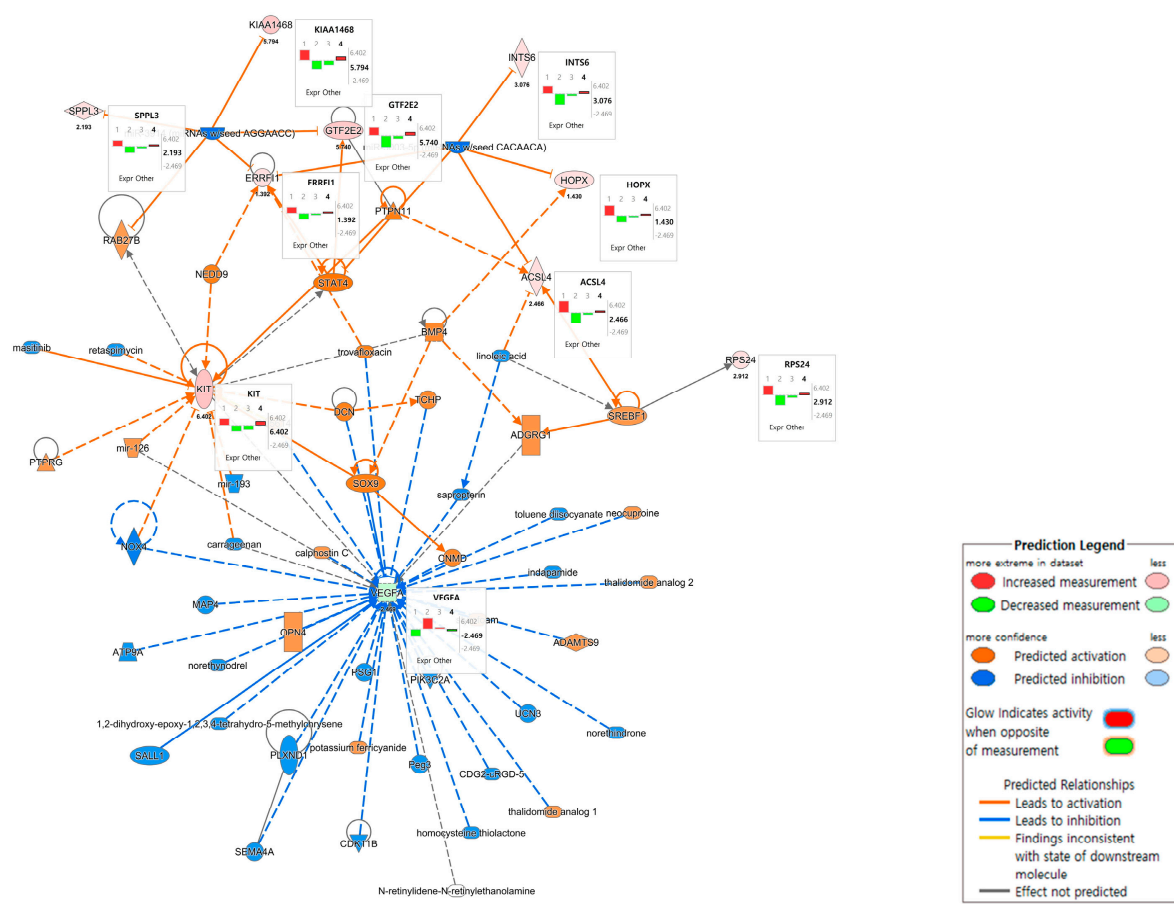

Figure 5. Gene networks associated with anti-asthma effects. Pathway depicts expression levels of anti-asthmatic genes regulated by DNA methylation changes in OVA vs. saline using IPA (A). Pathway depicts expression levels of anti-asthmatic genes regulated by DNA methylation changes in DSE vs. OVA (B). Bar plots next to genes in the network show changes in methylation and expression levels under each condition. The left panel of the network shows the bar plot legend, and the right panel provides a legend of the IPA network. 
Table 1. Candidate genes of integrative pattern analysis.

\begin{tabular}{|c|c|c|c|c|c|c|c|c|c|c|c|}
\hline \multirow{3}{*}{ Gene } & \multirow{3}{*}{ Pattern } & \multirow{3}{*}{ Transcript Name } & \multirow{3}{*}{ DMR Position } & \multicolumn{4}{|c|}{ Methylation } & \multicolumn{4}{|c|}{ Expression } \\
\hline & & & & \multicolumn{2}{|c|}{ OVA vs. Saline } & \multicolumn{2}{|c|}{ DSE vs. OVA } & \multicolumn{2}{|c|}{ OVA vs. Saline } & \multicolumn{2}{|c|}{ DSE vs. OVA } \\
\hline & & & & Delta-Beta & $p$-Value & Delta-Beta & $p$-Value & Fold Change & $p$-Value & Fold Change & $p$-Value \\
\hline 2310035C23Rik & Pattern A & NM_173187 & chr1 $\$ 105680001 \$ 105681000$ & 17.91792 & 0.001044 & -14.3229 & 0.000793 & -7.15782 & 0.003233 & 5.794239 & 0.01018 \\
\hline 2310035C23Rik & Pattern A & NM_173187 & chr1\$105680501\$105681500 & 17.91792 & 0.001044 & -14.6318 & 0.000691 & -7.15782 & 0.003233 & 5.794239 & 0.01018 \\
\hline Acsl4 & Pattern A & NM_019477 & chrX\$142468501\$142469500 & 20.1579 & $4.01 \times 10^{-8}$ & -18.5894 & $3.60 \times 10^{-9}$ & -3.31049 & 0.008914 & 2.466142 & 0.036953 \\
\hline Clec4d & Pattern A & NM_010819 & chr6\$123258501\$123259500 & 13.24306 & $3.05 \times 10^{-6}$ & -12.7135 & $8.06 \times 10^{-8}$ & -1.8897 & 0.031682 & 1.659789 & 0.047727 \\
\hline$D \lg 3$ & Pattern A & NM_001177780 & chrX\$100801001\$100802000 & 21.42624 & $2.03 \times 10^{-5}$ & -15.8962 & 0.000187 & -3.39981 & 0.001902 & 2.512896 & 0.013947 \\
\hline Errfi1 & Pattern A & NM_133753 & chr $4 \$ 150851001 \$ 150852000$ & 10.49137 & $6.31 \times 10^{-5}$ & -10.8417 & $1.84 \times 10^{-6}$ & -2.72862 & 0.000324 & 1.392076 & 0.044534 \\
\hline Gpr155 & Pattern A & NM_001190297 & chr2\$73402501\$73403500 & 14.45583 & 0.001214 & -13.4888 & 0.000127 & -2.73567 & 0.000478 & 1.421815 & 0.047225 \\
\hline Gtf2e2 & Pattern A & NM_026584 & chr8 $\$ 33705501 \$ 33706500$ & 14.15904 & $2.14 \times 10^{-7}$ & -11.7841 & $8.08 \times 10^{-8}$ & -4.88319 & 0.011646 & 5.739732 & 0.003803 \\
\hline Gtf2e2 & Pattern A & NM_026584 & chr8\$33721001\$33722000 & 17.11279 & 0.000173 & -19.3001 & $6.24 \times 10^{-8}$ & -4.88319 & 0.011646 & 5.739732 & 0.003803 \\
\hline Gtf2e2 & Pattern A & NM_026584 & chr8\$33721501\$33722500 & 14.40352 & 0.000466 & -20.3312 & $8.98 \times 10^{-12}$ & -4.88319 & 0.011646 & 5.739732 & 0.003803 \\
\hline Hopx & Pattern A & NM_001159900 & chr5\$77114001\$77115000 & 17.89375 & $2.10 \times 10^{-9}$ & -11.912 & $5.71 \times 10^{-6}$ & -2.37969 & 0.00221 & 1.429905 & 0.04741 \\
\hline Ints6 & Pattern A & NM_008715 & chr14\$62774001\$62775000 & 12.51032 & 0.0003 & -18.3492 & $3.51 \times 10^{-9}$ & -3.22793 & 0.001325 & 3.076277 & 0.001547 \\
\hline Kat $6 a$ & Pattern A & NM_001081149 & chr8 $\$ 22873001 \$ 22874000$ & 21.42651 & 0.002265 & -40.3226 & $7.75 \times 10^{-14}$ & -2.32499 & 0.028763 & 3.024011 & 0.004454 \\
\hline Kit & Pattern A & NM_001122733 & chr5\$75579001\$75580000 & 11.51523 & $7.89 \times 10^{-5}$ & -10.3308 & $3.31 \times 10^{-5}$ & -7.78059 & 0.000103 & 6.402056 & 0.00061 \\
\hline Kit & Pattern A & NM_001122733 & chr5\$75579501\$75580500 & 10.35753 & $1.89 \times 10^{-6}$ & -11.8328 & $2.81 \times 10^{-10}$ & -7.78059 & 0.000103 & 6.402056 & 0.00061 \\
\hline Ndel1 & Pattern A & NM_023668 & chr11\$68825001\$68826000 & 21.79487 & 0.000464 & -22.6016 & $3.14 \times 10^{-5}$ & -4.91466 & 0.000645 & 4.250178 & 0.001895 \\
\hline Pcmtd1 & Pattern A & NM_183028 & chr1 $\$ 7543001 \$ 7544000$ & 10.6038 & 0.001635 & -11.7335 & $2.64 \times 10^{-5}$ & -5.37129 & $3.98 \times 10^{-7}$ & 2.821372 & 0.002068 \\
\hline$R p s 24$ & Pattern A & NM_011297 & chr14\$24497001\$24498000 & 17.13836 & 0.000641 & -17.2688 & 0.00011 & -4.04976 & 0.001056 & 2.911984 & 0.01074 \\
\hline$R p s 24$ & Pattern A & NM_207635 & chr14\$24497001\$24498000 & 17.13836 & 0.000641 & -17.2688 & 0.00011 & -4.00879 & 0.000414 & 3.224884 & 0.002518 \\
\hline$R p s 24$ & Pattern A & NM_011297 & chr14\$24497501\$24498500 & 17.13836 & 0.000641 & -17.2688 & 0.00011 & -4.04976 & 0.001056 & 2.911984 & 0.01074 \\
\hline Rps24 & Pattern A & NM_207635 & chr14\$24497501\$24498500 & 17.13836 & 0.000641 & -17.2688 & 0.00011 & -4.00879 & 0.000414 & 3.224884 & 0.002518 \\
\hline Shroom 2 & Pattern A & NM_001290684 & chrX $\$ 152750501 \$ 152751500$ & 19.51472 & $1.06 \times 10^{-5}$ & -16.7372 & $4.44 \times 10^{-6}$ & -11.7053 & $2.06 \times 10^{-6}$ & 9.44746 & 0.00004 \\
\hline Shroom 2 & Pattern A & NM_001290684 & chrX\$152751001\$152752000 & 19.51472 & $1.06 \times 10^{-5}$ & -16.7372 & $4.44 \times 10^{-6}$ & -11.7053 & $2.06 \times 10^{-6}$ & 9.44746 & 0.00004 \\
\hline Sppl3 & Pattern A & NM_029012 & chr5\$115027001\$115028000 & 10.11934 & $9.88 \times 10^{-7}$ & -11.4787 & $2.84 \times 10^{-11}$ & -2.95759 & 0.000555 & 2.19341 & 0.006261 \\
\hline Zfp568 & Pattern A & NM_001167873 & chr7\$30013001\$30014000 & 10.90426 & 0.00247 & -17.1518 & $1.59 \times 10^{-7}$ & -4.33247 & 0.007645 & 4.172162 & 0.008244 \\
\hline Vegfa & Pattern B & NM_001025257 & chr17\$46024501\$46025500 & -13.0325 & 0.004499 & 18.71345 & $3.94 \times 10^{-6}$ & 2.522877 & 0.014275 & -2.46869 & 0.007728 \\
\hline Vegfa & Pattern B & NM_001025257 & chr17\$46025001\$46026000 & -13.0325 & 0.004499 & 18.71345 & $3.94 \times 10^{-6}$ & 2.522877 & 0.014275 & -2.46869 & 0.007728 \\
\hline
\end{tabular}


Figure $5 \mathrm{~B}$ shows the predicted pathways in DSE vs. OVA. The majority of genes, including Kit, Acsl4, Hopx, Rps24, Errfi1, Gtf2e2, Ints6, 2310035C23Rik (KIAA1468) and Sppl3, were upregulated; only Vegfa was downregulated; and the methylation changes were the opposite of those observed in OVA vs. saline. Two hub genes (Vegfa and Kit) in these integrated networks exhibited an inverse correlation between DNA methylation and gene expression. Hub genes, which are highly interconnected nodes in IPA modules, are functionally significant. Thus, Vegfa (vascular endothelial growth factor A) and Kit (proto-oncogene receptor tyrosine kinase) were identified as the main regulators of the integrated network, potentially explaining the differential regulation of their downstream target genes among the control, OVA-induced and DSE-treated groups.

\section{Discussion}

The aim of this study was to discover distinct differential methylomic signatures in lung tissue samples obtained from an asthma-induced mouse model. The results of H\&E staining and BALF analysis confirmed that DSE inhibited allergic lung inflammation by decreasing inflammatory cell infiltration and IL-4 production (Figure 2). Therefore, we investigated the possibility that DSE could be used as a treatment for asthma. To understand how asthmatic inflammation (OVA-induced) and anti-asthmatic effects (DSE-treated) regulate respiratory disease, and how to determine which genes cause disease, we compared differences among saline, OVA-induced and DSE-treated mice by RNA-seq and Methyl-seq. Previous studies that investigated the epigenetic mechanisms underlying asthma [3,21] and characterized the anti-asthmatic effects [22-26] partly explained the epigenetic regulation of anti-asthmatic effects by herbal medicine. However, the results of our study provide an integrated perspective on the epigenetic changes involved in the anti-asthmatic effects of $D$. sophia seeds, as determined by genome-wide gene expression and methylation profiling. This is the first integrated study of the role of DNA methylation and gene expression in the anti-asthmatic effects of $D$. sophia seeds. Our findings effectively identify key genes underlying anti-asthmatic patterns and elucidate an integrated network related to asthma.

To identify anti-asthmatic changes in methylation, we profiled genome-wide methylation patterns, including the hyper/hypo and hypo/hyper patterns described in the Results section, during OVA induction followed by treatment with a medicinal herb. Gene Ontology (GO) analysis of these candidate genes revealed strong enrichment of terms related to olfactory disorders, myeloid cells, osteoclasts and cytokine/chemokine signaling pathways. Although asthma has various causes, airway inflammation is a common triggering mechanism [27-30]. Airway inflammation is mediated by chemokines and cytokines [31-33], suggesting that changes in the methylation of genes associated with cytokines/chemokines play an important role in the onset of asthma. Additionally, we also observed enrichment in ECM-receptor interaction and neurotrophin signaling (Table S4). ECM-receptors have direct and indirect effects on cell regulation and interact with eosinophilic asthma [10,34], whereas growth factors such as neurotrophins and their receptors are important in normal lung development, developmental lung disease, allergies and inflammatory conditions such as rhinitis or asthma [35-39].

By combining Methyl-seq with RNA-seq, we identified 18 candidate genes for which DNA methylation changed as a result of the anti-asthmatic effects of DSE. GO analysis revealed that genes whose expression returned to control levels upon DSE treatment were enriched in asthma-related functions such as lung alveolus development, lung vasculature development, and vasculature development.

In addition, we identified highly connected hub genes in integrated networks of DNA methylation and gene expression. Notably, Vegfa was regulated in an "up/down" manner, due to its hypo/hyper methylation pattern, in OVA-induced vs. saline and DSE-treated vs. OVA-induced mice. $V e g f a$, a member of the vascular endothelial growth factor family, promotes allergic inflammation and plays an important role in Th2 inflammation [40]. Furthermore, several studies have reported that Vegfa levels are increased in tissues and biological samples from asthma patients [40-42]. Kit, which was regulated in a "down/up" fashion, due to its hyper/hypo methylation pattern, is related to the NF- $\mathrm{KB}$ 
signaling pathway, which regulates pro-inflammatory mediators [43], and may help to maintain alveolar structure [44]. These results indicate that methylation changes of two hub genes play important roles in regulating the anti-inflammatory effect. We also identify previously unreported genes with concordant molecular changes in asthma: Shroom2, Pcmtd1, Zfp568 and 2310035C23Rik were significantly down/upregulated and hyper/hypo methylated.

This study had several limitations, including the fact that Methyl-seq technology was used to profile the methylome without any investigation of histone modifications, e.g., by ChIP-seq. In various omics analyses, it is important to keep in mind that expression of a candidate gene could be affected by methylation changes in a distal enhancer, or that a given gene could contain an enhancer region for other genes. Further work will be needed to examine changes in methylation at distal enhancer regions. Other omics studies such as single-cell or DNA resources based approaches also will be needed for identification of various causes. Moreover, larger sample sizes will be required for a more powerful characterization of the epigenetic mechanisms involved in restoration of control levels (of methylation or gene expression) by DSE treatment.

\section{Materials and Methods}

\subsection{Plant Material and Preparation of DSE}

Seeds of D. sophia were collected by Dr. Sungyu Yang and Dr. Byeong Cheol Moon of the Korea Institute of Oriental Medicine (KIOM) in Geumsan, Chungcheongnam-do, Korea. The plants used in this study were authenticated based on macroscopic morphological characteristics by Dr. Goya Choi of KIOM. The voucher specimens were deposited in the Korean Herbarium of Standard Herbal Resources (herbarium code KIOM, Specimen 2-18-0137) at the KIOM. Dried seeds of D. sophia (760 g) were extracted twice with $70 \%$ ethanol (with $3 \mathrm{~h}$ reflux) at $80^{\circ} \mathrm{C}$, and the extract was concentrated under reduced pressure. Yields of DSE were approximately $7.44 \%(w t / w t)$.

\subsection{In Vivo Experiments}

\subsubsection{Animals}

Eight-week-old female BALB/c mice were purchased from Daehan BioLink Co., Ltd. (Chungcheongbuk-do, Korea) and housed under specific pathogen-free conditions with freely available food and water. All animal care and experimental procedures were performed with the approval of the Institutional Animal Care and Use Committee (IACUC) of the KIOM (17-073).

\subsubsection{Induction of Lung Inflammation and the Administration of Drug}

Asthma was induced as previously described with some modifications [45,46]. To induce allergic lung inflammation, mice were sensitized with OVA twice, 7 days apart (i.e., on Days 0 and 7) by intraperitoneal injection of OVA $(50 \mu \mathrm{g})$ emulsified with $200 \mu \mathrm{L}$ of aluminum sulfate (Alum; InvivoGen, San Diego, CA, USA). Beginning on Day 12, the mice were challenged with $50 \mu \mathrm{L}$ of OVA (25 $\mu \mathrm{g})$ intranasally for 4 days under anesthesia with $2 \%$ isoflurane (Piramal Critical Care Inc., Bethlehem, PA, USA) delivered by a Vevo ${ }^{\mathrm{TM}}$ Compact Anesthesia System (FUJIFILM VisualSonics, Toronto, ON, Canada). The mice were divided into four groups ( $n=7$ per group). One was injected with saline only, and the remaining three groups were sensitized to OVA. On Days 9-15, the treatment groups received oral administration of vehicle, DSE $(200 \mathrm{mg} / \mathrm{kg})$ for 7 consecutive days.

\subsubsection{Histological Analysis}

For euthanasia, an overdose of pentobarbital sodium (a short-acting barbiturate) was injected in accordance with IACUC guidelines. Lung tissues of mice were collected and fixed in $10 \%$ formalin. Fixed tissues were embedded in paraffin and cut into $5-\mu \mathrm{m}$ sections with a microtome 
(Leica, Nussloch, Germany). The sections were deparaffinized and stained with H\&E for analysis of inflammatory changes.

4.2.4. Collection of BALF, Quantification of Inflammatory Cells and Measurement of IL-4 Levels in BALF

BALF was collected by endotracheal lavage with $1 \mathrm{~mL}$ of Dulbecco's phosphate-buffered saline (ScienCell Research Laboratories, Carlsbad, CA, USA) via intubation. To determine differential cell counts in the BALF, cells were placed on a slide by cytospin and then stained with Hema 3 solution (Fisher HealthCare, Pittsburgh, PA, USA). The proportion of each cell type was determined based on morphology, and the number of cells was calculated by multiplying the proportions by the total cell count. To measure cytokine levels, BALF was centrifuged for $5 \mathrm{~min}$ at $4{ }^{\circ} \mathrm{C}$, and then the levels of IL-4 were measured in the supernatant by an ELISA, as previously described [47]. Briefly, 11B11 antibody was used to capture IL-4, and BVD6-24G2 biotinylated antibody was used for its detection. Antibodies were purchased from BD Biosciences (San Diego, CA, USA).

\subsection{Methyl-Seq and Data Analysis}

SureSelect Methylation libraries were prepared according to the manufacturer's instructions (Agilent Methyl-seq protocol). Three micrograms of high-quality genomic DNA were diluted with $50 \mu \mathrm{L}$ EB buffer and fragmented to a median size of $150 \mathrm{bp}$ using the Covaris-S2 instrument (Covaris, Woburn, MA, USA). Following successful shearing, the gDNA was subjected to end blunting, $\mathrm{dA}$-tailing and the ligation with methylated adapters. The constructed libraries for the capture were quantified using the Quant-iT dsDNA HS Assay Kit (Invitrogen, Carlsbad, CA, USA) with the Invitrogen Qubit fluorometer (Invitrogen, Carlsbad, CA, USA). The hybridizations were performed using a thermal cycler that was set at $65^{\circ} \mathrm{C}$ for $24 \mathrm{~h}$ with the lid heated to $105^{\circ} \mathrm{C}$; then, Dynabeads ${ }^{\circledR}$ MyOne Streptavidin T1 (Invitrogen, Carlsbad, CA, USA) was used to capture the biotinylated RNA probes. Finally, the captured library samples were washed and eluted for the bisulfite conversion. Eluted DNA was bisulfite converted using the EZ DNA Methylation-Gold kit (ZymoResearch, Cat. \#D5005, Carlsbad, CA, USA), following the Agilent instruction manual. Furthermore, the treated DNA was amplified by PCR, converting uracil residues in the sample to thymidine. Amplified bisulfied-treated libraries were purified using AMPure XP beads (Beckman Coulter, High Wycombe, UK). To index the captured libraries, bisulfied-treated libraries were amplified by PCR. Indexed libraries were purified and accessed by Agilent 2100 Bioanalyzer (High Sensitivity DNA assay, Agilent, Santa Clara, CA, USA). Sequencing runs were performed in paired-end mode using the HiSeq 2500 platforms (Illumina, San Diego, CA, USA). Using the TruSeq SBS Kits v4 (Illumina, San Diego, CA, USA) for the HiSeq, sequencing-by-synthesis reactions were extended for 101 cycles from each end.

After sequencing, the reads were trimmed to remove adapters and low-quality reads (per-base quality < 20) and thereby improve paired-end mapping using Cutadapt (v. 1.13) [48]. Reads were mapped to the Mus musculus genome (mm10) with Bismark (v. 0.17.0) [49]. The methylKit R package (v. 1.6.0) [50] was used for analysis of Methyl-seq data and DMR identification (window size: 1000 and step size: 500). Regions with two or more CpGs, $\geq 10 \%$ mean methylation difference and $q$-value $<0.01$ were considered significant. methylKit software (v. 1.9.0) was used for annotation of peaks and assessment of the distribution of methylation peaks across genomic features. Genomic features were classified into six types of regions (intergenic, $5^{\prime}$ UTR, $3^{\prime}$ UTR, promoter, CDS and intron) based on the UCSC genome annotation.

\subsection{RNA-Seq and Data Analysis}

Total RNA was also extracted from lung tissues used in the methyl-seq using Nucleo spin RNA kit (MACHREY-NAGEL, Dueren, Germany). The quantity and quality of the total RNA were evaluated using Agilent 2100 Bioanalyzer (Agilent, Santa Clara, CA, USA) and assessing the RNA quality indicator (RIN). Library preparation was performed with an Illumina TruSeq Stranded mRNA sample 
preparation kit (Illumina, San Diego, CA, USA) according to the manufacturer's instructions. Indexing adapters were ligated to the ends of the cDNA fragments using ligation mix reagent at $30{ }^{\circ} \mathrm{C}$ for $10 \mathrm{~min}$. After twice washing with sample purification bead, PCR was performed to enrich those cDNA fragments that have adapter molecules on both ends. Thermocycler conditions were as follows: $98^{\circ} \mathrm{C}$ for $30 \mathrm{~s} ; 15$ cycles of $98{ }^{\circ} \mathrm{C}$ for $10 \mathrm{~s}, 60^{\circ} \mathrm{C}$ for $30 \mathrm{~s}$, and $72{ }^{\circ} \mathrm{C}$ for $30 \mathrm{~s}$; and a final extension at $72{ }^{\circ} \mathrm{C}$ for $5 \mathrm{~min}$. Finally, quality and band size of libraries were assessed using Agilent 2100 Bioanalyzer. Libraries were quantified by qPCR using CFX96 Real Time System (Biorad, Hercules, CA, USA). After normalization, sequencing of the prepared library was conducted on the Nextseq 500 (Illumina, San Diego, CA, USA) with 76 bp paired-end reads.

Reads were trimmed to remove adapters and low-quality reads (per-base quality $<20$ ) and thereby improve paired-end mapping. High-quality sequence reads were mapped to the Mus musculus genome (mm10) using Hisat2 (v. 2.1.0) [51], and gene expression levels were quantified with the ballgown R package (v. 2.12.0) [52]. Genes differentially expressed among the three groups were evaluated by the edgeR package (v. 3.0.8) [53], which is based on negative binomial models for RNA-seq count data. Differentially expressed genes were screened with a cutoff threshold of log (FC) $\geq|1|$ and $p$-value $<0.05$.

\subsection{Correlation Analysis between DNA Methylation and Expression}

To identify genes with an inverse relationship between expression and methylation, the expression level was adjusted to a range from 0 to 1 , as for methylation, and then Spearman rank correlations were used to assess the relationship between $\mathrm{CpG}$ methylation and gene expression. To find the functional mechanisms involving these genes, we conducted GO term and KEGG pathway analysis using DAVID (v. 6.7) [54], which provides a set of data-mining tools that systematically combine functionally descriptive data with intuitive graphical displays.

\subsection{Pathway Analysis}

IPA (v. 1.13, Qiagen, Valencia, CA, USA) software was used to predict the networks containing the 18 candidate genes whose expression changed due to alterations in DNA methylation in response to anti-asthmatic treatment. First, we used network connection tools in IPA to display known functional interactions between these genes, and then further integrated our findings by overlaying genes involved in asthma, lung development and immune disease. To determine how each gene affects the integrated network, we overlaid the fold change in gene expression and DNA methylation $\Delta \beta$ values. In the figures, bar plots beside the genes in the network show DNA methylation and expression change under each condition. Molecules are represented as nodes, and the biological relationships between nodes are represented as edges (line). The intensity of node color indicates the degree of expression change.

\subsection{Data Access}

The data generated over the course of this study have been deposited into the Gene Expression Omnibus (GEO) under accession number GSE114587 (https: / / www.ncbi.nlm.nih.gov/geo/query/acc. cgi?acc=GSE114587; secure token for reviewer: itytgkuoptclnej).

\section{Conclusions}

In summary, the integrative approach described here identified anti-asthmatic changes in a mouse model, identifying 18 genes and integrated networks involved in methylation and gene expression. We identified several epigenetically regulated genes and pathways involved in anti-asthmatic effects, which should facilitate development of novel and effective therapeutic agents for asthma. 
Supplementary Materials: The following are available online, Figure S1: Workflow of integrated profiling, Figure S2: Experimental scheme and sample information, Figure S3: Histogram for percent methylation distribution, Figure S4: Histogram for the read coverage, Figure S5: Correlation plot between methylation and expression of candidate genes, Table S1: DMR list in OVA vs. Saline, Table S2: DMR list in DSE vs. OVA, Table S3: DMR list in anti-asthmatic patterns, Table S4: DAVID result in hyper/hypo pattern, Table S5: DAVID result in hypo/hyper pattern, Table S6: DAVID result in 18 candidate genes, Table S7: Network genes and chemicals associated with asthma.

Author Contributions: S.-J.B. contributed to the design, analyzed the data, and drafted the manuscript; J.M.C., Y.-S.S. and S.-B.K. conducted the in vivo experiments, data interpretation and wrote the manuscript related content; T.-W.K. and G.-H.S. conducted the network analysis and data interpretation; B.S. and Y.J. conducted the research of previous studies; and C.K. contributed to the direction and concept of the experiment. All authors reviewed the manuscript.

Funding: This research was supported by the Maximize utilization of knowledge about herbal resource (grant number: K18404) and funded by the Korea Institute of Oriental Medicine.

Conflicts of Interest: The authors declare no conflict of interest.

\section{References}

1. Salam, M.T.; Zhang, Y.; Begum, K. Epigenetics and childhood asthma: Current evidence and future research directions. Epigenomics 2012, 4, 415-429. [CrossRef] [PubMed]

2. Chogtu, B.; Bhattacharjee, D.; Magazine, R. Epigenetics: The new frontier in the landscape of asthma. Scientifica 2016, 2016. [CrossRef] [PubMed]

3. Yang, I.V.; Schwartz, D.A. Epigenetic mechanisms and the development of asthma. J. Allergy Clin. Immunol. 2012, 130, 1243-1255. [CrossRef] [PubMed]

4. Begin, P.; Nadeau, K.C. Epigenetic regulation of asthma and allergic disease. Allergy Asthma Clin. Immunol. 2014, 10. [CrossRef] [PubMed]

5. Kuo, C.H.; Hsieh, C.C.; Lee, M.S.; Chang, K.T.; Kuo, H.F.; Hung, C.H. Epigenetic regulation in allergic diseases and related studies. Asia Pac. Allergy 2014, 4, 14-18. [CrossRef] [PubMed]

6. Gao, L.; Millstein, J.; Siegmund, K.D.; Dubeau, L.; Maguire, R.; Gilliland, F.D.; Murphy, S.K.; Hoyo, C.; Breton, C.V. Epigenetic regulation of $A X L$ and risk of childhood asthma symptoms. Clin. Epigenet. 2017, 9. [CrossRef] [PubMed]

7. Nicodemus-Johnson, J.; Myers, R.A.; Sakabe, N.J.; Sobreira, D.R.; Hogarth, D.K.; Naureckas, E.T.; Sperling, A.I.; Solway, J.; White, S.R.; Nobrega, M.A.; et al. DNA methylation in lung cells is associated with asthma endotypes and genetic risk. JCI Insight 2016, 1, e90151. [CrossRef] [PubMed]

8. Xu, C.J.; Soderhall, C.; Bustamante, M.; Baiz, N.; Gruzieva, O.; Gehring, U.; Mason, D.; Chatzi, L.; Basterrechea, M.; Llop, S.; et al. DNA methylation in childhood asthma: An epigenome-wide meta-analysis. Lancet Respir. Med. 2018, 6, 379-388. [CrossRef]

9. Zhang, X.; Biagini Myers, J.M.; Yadagiri, V.K.; Ulm, A.; Chen, X.; Weirauch, M.T.; Khurana Hershey, G.K.; Ji, H. Nasal DNA methylation differentiates corticosteroid treatment response in pediatric asthma: A pilot study. PLoS ONE 2017, 12, e0186150. [CrossRef] [PubMed]

10. Rajendran, P.; Ho, E.; Williams, D.E.; Dashwood, R.H. Dietary phytochemicals, HDAC inhibition, and DNA damage/repair defects in cancer cells. Clin. Epigenet. 2011, 3. [CrossRef] [PubMed]

11. Hernandez-Ledesma, B.; Hsieh, C.C.; Martinez-Villaluenga, C. Food bioactive compounds against diseases of the 21st century 2016. Biomed. Res. Int. 2017, 2017. [CrossRef] [PubMed]

12. Park, H.S.; Kim, S.R.; Kim, J.O.; Lee, Y.C. The roles of phytochemicals in bronchial asthma. Molecules 2010, 15, 6810-6834. [CrossRef] [PubMed]

13. HadiNezhad, M.; Rowland, O.; Hosseinian, F. The fatty acid profile and phenolic composition of Descurainia sophia seeds extracted by supercritical $\mathrm{CO}_{2}$. J. Am. Oil Chem. Soc. 2015, 92, 1379-1390. [CrossRef]

14. Rahman, M.J.; Costa de Camargo, A.; Shahidi, F. Phenolic profiles and antioxidant activity of defatted camelina and sophia seeds. Food Chem. 2018, 240, 917-925. [CrossRef] [PubMed]

15. Korea Institute of Oriental Medicine. Defining Dictionary for Medicinal Herbs [Korean, 'Hanyak Giwon Sajeon']; Korea Institute of Oriental Medicine. Available online: http://boncho.kiom.re.kr/codex/ (accessed on 16 April 2018). 
16. Kim, B.Y.; Lee, J.; Park, S.J.; Bang, O.S.; Kim, N.S. Gene expression profile of the A549 human non-small cell lung carcinoma cell line following treatment with the seeds of Descurainia sophia, a potential anticancer drug. Evid. Based Complement. Altern. Med. 2013. [CrossRef] [PubMed]

17. Lee, Y.J.; Kim, N.S.; Kim, H.; Yi, J.M.; Oh, S.M.; Bang, O.S.; Lee, J. Cytotoxic and anti-inflammatory constituents from the seeds of Descurainia sophia. Arch. Pharm. Res. 2013, 36, 536-541. [CrossRef] [PubMed]

18. Khodarahmi, E.; Asghari, G.H.; Hassanzadeh, F.; Mirian, M.; Khodarahmi, G.A. Cytotoxic evaluation of volatile oil from Descurainia sophia seeds on MCF-7 and HeLa cell lines. Res. Pharm. Sci. 2015, 10, 169-176. [PubMed]

19. Park, J.S.; Lim, C.J.; Bang, O.S.; Kim, N.S. Ethanolic extract of Descurainia sophia seeds sensitizes A549 human lung cancer cells to TRAIL cytotoxicity by upregulating death receptors. BMC Complement. Altern. Med. 2016, 16. [CrossRef] [PubMed]

20. Gour, N.; Wills-Karp, M. IL-4 and IL-13 signaling in allergic airway disease. Cytokine 2015, 75, 68-78. [CrossRef] [PubMed]

21. Perry, M.M.; Lavender, P.; Kuo, C.S.; Galea, F.; Michaeloudes, C.; Flanagan, J.M.; Fan Chung, K.; Adcock, I.M. DNA methylation modules in airway smooth muscle are associated with asthma severity. Eur. Respir. J. 2018, 51. [CrossRef] [PubMed]

22. Epstein, M. The calcium antagonist controversy: The emerging importance of drug formulation as a determinant of risk. Am. J. Cardiol. 1997, 79, 9-19. [CrossRef]

23. Montrose, L.; Ward, T.J.; Semmens, E.O.; Cho, Y.H.; Brown, B.; Noonan, C.W. Dietary intake is associated with respiratory health outcomes and DNA methylation in children with asthma. Allergy Asthma Clin. Immunol. 2017, 13. [CrossRef] [PubMed]

24. Lee, M.; Kim, S.; Kwon, O.K.; Oh, S.R.; Lee, H.K.; Ahn, K. Anti-inflammatory and anti-asthmatic effects of resveratrol, a polyphenolic stilbene, in a mouse model of allergic asthma. Int. Immunopharmacol. 2009, 9, 418-424. [CrossRef] [PubMed]

25. Boskabady, M.H.; Mohsenpoor, N.; Takaloo, L. Antiasthmatic effect of Nigella sativa in airways of asthmatic patients. Phytomedicine 2010, 17, 707-713. [CrossRef] [PubMed]

26. Yang, W.K.; Lee, C.H.; Kim, M.H.; Kim, S.H.; Choi, H.Y.; Yeo, Y.; Park, Y.C. Effects of inhalable microparticles of Seonpyejeongcheon-Tang in an asthma mouse model: Effects of Microparticles of SJT. J. Pharmacopunct. 2016, 19, 303-311. [CrossRef] [PubMed]

27. Busse, W.W.; Calhoun, W.F.; Sedgwick, J.D. Mechanism of airway inflammation in asthma. Am. Rev. Respir. Dis. 1993, 147, S20-S24. [CrossRef] [PubMed]

28. Murdoch, J.R.; Lloyd, C.M. Chronic inflammation and asthma. Mutat. Res. 2010, 690, 24-39. [CrossRef] [PubMed]

29. Nakagome, K.; Nagata, M. Pathogenesis of airway inflammation in bronchial asthma. Auris Nasus Larynx 2011, 38, 555-563. [CrossRef] [PubMed]

30. Sugita, M.; Kuribayashi, K.; Nakagomi, T.; Miyata, S.; Matsuyama, T.; Kitada, O. Allergic bronchial asthma: Airway inflammation and hyperresponsiveness. Intern. Med. 2003, 42, 636-643. [CrossRef] [PubMed]

31. Aisenberg, W.H.; Huang, J.; Zhu, W.; Rajkumar, P.; Cruz, R.; Santhanam, L.; Natarajan, N.; Yong, H.M.; De Santiago, B.; Oh, J.J.; et al. Defining an olfactory receptor function in airway smooth muscle cells. Sci. Rep. 2016. [CrossRef] [PubMed]

32. Wang, Y.; Bai, C.; Li, K.; Adler, K.B.; Wang, X. Role of airway epithelial cells in development of asthma and allergic rhinitis. Respir. Med. 2008, 102, 949-955. [CrossRef] [PubMed]

33. Little, P.J.; Martin, B.R. Cannabinoid modulation of cyclic AMP accumulation in synaptosomes. NIDA Res. Monogr. 1989, 95, 282-283. [PubMed]

34. Yin, L.M.; Jiang, G.H.; Wang, Y.; Wang, Y.; Liu, Y.Y.; Jin, W.R.; Zhang, Z.; Xu, Y.D.; Yang, Y.Q. Serial analysis of gene expression in a rat lung model of asthma. Respirology 2008, 13, 972-982. [CrossRef] [PubMed]

35. Prakash, Y.; Thompson, M.A.; Meuchel, L.; Pabelick, C.M.; Mantilla, C.B.; Zaidi, S.; Martin, R.J. Neurotrophins in lung health and disease. Expert Rev. Respir. Med. 2010, 4, 395-411. [CrossRef] [PubMed]

36. Barrios, J.; Ai, X. Neurotrophins in Asthma. Curr. Allergy Asthma Rep. 2018. [CrossRef] [PubMed]

37. Renz, H.; Kerzel, S.; Nockher, W.A. The role of neurotrophins in bronchial asthma: Contribution of the pan-neurotrophin receptor p75. Prog. Brain Res. 2004, 146, 325-333. [PubMed]

38. Rochlitzer, S.; Nassenstein, C.; Braun, A. The contribution of neurotrophins to the pathogenesis of allergic asthma. Biochem. Soc. Trans. 2006, 34, 594-599. [CrossRef] [PubMed] 
39. Renz, H. Neurotrophins in bronchial asthma. Respir. Res. 2001, 2, 265-268. [CrossRef] [PubMed]

40. Lee, C.G.; Ma, B.; Takyar, S.; Ahangari, F.; Delacruz, C.; He, C.H.; Elias, J.A. Studies of vascular endothelial growth factor in asthma and chronic obstructive pulmonary disease. Proc. Am. Thorac. Soc. 2011, 8, 512-515. [CrossRef] [PubMed]

41. Hoshino, M.; Nakamura, Y.; Hamid, Q.A. Gene expression of vascular endothelial growth factor and its receptors and angiogenesis in bronchial asthma. J. Allergy Clin. Immunol. 2001, 107, 1034-1038. [CrossRef] [PubMed]

42. Smith, R. Is VEGF a potential therapeutic target in asthma? Pneumologia 2014, 63, 197-199.

43. Song, L.; Martinez, L.; Zigmond, Z.M.; Hernandez, D.R.; Lassance-Soares, R.M.; Selman, G.; Vazquez-Padron, R.I. c-Kit modifies the inflammatory status of smooth muscle cells. Peer J. 2017, 5, e3418. [CrossRef] [PubMed]

44. Lindsey, J.Y.; Ganguly, K.; Brass, D.M.; Li, Z.; Potts, E.N.; Degan, S.; Chen, H.; Brockway, B.; Abraham, S.N.; Berndt, A.; et al. c-Kit is essential for alveolar maintenance and protection from emphysema-like disease in mice. Am. J. Respir. Crit. Care Med. 2011, 183, 1644-1652. [CrossRef] [PubMed]

45. Lee, A.R.; Chun, J.M.; Lee, A.Y.; Kim, H.S.; Gu, G.J.; Kwon, B.I. Reduced allergic lung inflammation by root extracts from two species of Peucedanum through inhibition of Th2 cell activation. J. Ethnopharmacol. 2017, 196, 75-83. [CrossRef] [PubMed]

46. Chun, J.M.; Lee, A.R.; Kim, H.S.; Lee, A.Y.; Gu, G.J.; Moon, B.C.; Kwon, B.I. Peucedanum japonicum extract attenuates allergic airway inflammation by inhibiting Th2 cell activation and production of pro-inflammatory mediators. J. Ethnopharmacol. 2018, 211, 78-88. [CrossRef] [PubMed]

47. Corry, D.B.; Rishi, K.; Kanellis, J.; Kiss, A.; Song Lz, L.Z.; Xu, J.; Feng, L.; Werb, Z.; Kheradmand, F. Decreased allergic lung inflammatory cell egression and increased susceptibility to asphyxiation in MMP2-deficiency. Nat. Immunol. 2002, 3, 347-353. [CrossRef] [PubMed]

48. Martin, M. Cutadapt removes adapter sequences from high-throughput sequencing reads. EMBnet. J. 2011, 17. [CrossRef]

49. Krueger, F.; Andrews, S.R. Bismark: A flexible aligner and methylation caller for Bisulfite-Seq applications. Bioinformatics 2011, 27, 1571-1572. [CrossRef] [PubMed]

50. Akalin, A.; Kormaksson, M.; Li, S.; Garrett-Bakelman, F.E.; Figueroa, M.E.; Melnick, A.; Mason, C.E. methylKit: A comprehensive R package for the analysis of genome-wide DNA methylation profiles. Genome Biol. 2012, 13, 87. [CrossRef] [PubMed]

51. Pertea, M.; Kim, D.; Pertea, G.M.; Leek, J.T.; Salzberg, S.L. Transcript-level expression analysis of RNA-seq experiments with HISAT, StringTie and Ballgown. Nat. Protoc. 2016, 11, 1650-1667. [CrossRef] [PubMed]

52. Frazee, A.C.; Pertea, G.; Jaffe, A.E.; Langmead, B.; Salzberg, S.L.; Leek, J.T. Flexible isoform-level differential expression analysis with Ballgown. BioRxiv 2014. [CrossRef]

53. Robinson, M.D.; McCarthy, D.J.; Smyth, G.K. edgeR: A Bioconductor package for differential expression analysis of digital gene expression data. Bioinformatics 2010, 26, 139-140. [CrossRef] [PubMed]

54. Huang, D.W.; Sherman, B.T.; Tan, Q.; Collins, J.R.; Alvord, W.G.; Roayaei, J.; Stephens, R.; Baseler, M.W.; Lane, H.C.; Lempicki, R.A. The DAVID Gene Functional Classification Tool: A novel biological module-centric algorithm to functionally analyze large gene lists. Genome Biol. 2007, 8, R183. [CrossRef] [PubMed]

Sample Availability: Not available. 